\title{
A Method for Feature Detection in Binary Tomography
}

\author{
Wagner Fortes ${ }^{1,2}$ and K. Joost Batenburg ${ }^{1,3}$ \\ 1 Centrum Wiskunde \& Informatica, Amsterdam, The Netherlands \\ 2 Mathematical Institute, Leiden University, The Netherlands \\ 3 Vision Lab, University of Antwerp, Belgium \\ \{wagner.fortes, joost. batenburg\}@cwi.nl
}

\begin{abstract}
Binary tomography deals with the problem of reconstructing a binary image from its projections. Depending on properties of the unkown original image, the constraint that the image is binary enables accurate reconstructions from a relatively small number of projection angles. Even in cases when insufficient information is available to compute an accurate reconstruction of the complete image, it may still be possible to determine certain features of it, such as straight boundaries, or homogeneous regions. In this paper, we present a computational technique for discovering the possible presence of such features in the unknown original image. We present numerical experiments, showing that it is often possible to accurately identify the presence of certain features, even without a full reconstruction.
\end{abstract}

\section{Introduction}

Binary tomography deals with the problem of reconstructing a binary image from its projections. While accurate image reconstruction requires availability of a large number of projections for general grey scale images, knowledge about the fact the the unknown original image is binary can drastically reduce the number of projection angles needed for a detailed reconstruction in some cases.

A range of algorithms have been proposed for binary tomography [1 $[4]$. Although each of these methods has demonstrated the ability to compute accurate reconstructions from a small number of projections in certain cases, none of these methods offer a guarantee that the reconstructed image is identical, or even similar to the unknown original image. In fact, one can state that giving such a guarantee will be impossible in general, as the reconstruction problem in binary tomography is known to be inherently unstable: a small change in the projection data can lead to a dramatic change in the (unique) reconstruction [5 7]. Moreover, several constructions are known for so-called switching components: binary images in which a selected set of zeros and ones can be interchanged, leading to a different image having the same projections [8, 9].

Even in cases when insufficient information is available to compute an accurate reconstruction of the complete image, it may still be possible to answer certain questions about the original image, or to determine certain features of it.

R. Gonzalez-Diaz, M.-J. Jimenez, B. Medrano (Eds.): DGCI 2013, LNCS 7749, pp. 372-382, 2013.

(C) Springer-Verlag Berlin Heidelberg 2013 
In [10], it was shown that connectivity and convexity properties can be derived - to some extent - directly from the projection data. It can also be desirable to know whether a certain boundary or homogeneous region can possibly exist in the unkown image, or not.

Even though finding a binary solution of the reconstruction problem is typically hard, it is often easier to prove that a solution cannot exist. For example, if the projections do not satisfy certain consistency conditions, a solution will certainly not exist. General consistency conditions for the Radon transform are presented in [11], while a detailed analysis of consistency conditions for the grid model in discrete tomography can be found in [12]. A particular condition for the existence of binary solutions is given in [13], which will be used and extended throughout the present paper.

In this article, we extend the general idea of consistency to the detection whether or not certain substructures can exist in the original image. We present a computational technique for discovering the possible presence of certain features (e.g., blobs, edges). For each feature, a probe structure is defined, which can detect that particular feature. Based on an analysis of the existence of binary solutions of the reconstruction problem, our technique can prove, in certain cases, if the probed feature cannot exist in a given region of the original image. Our approach is independent of a particular reconstructed image or reconstruction method.

This paper is structured as follows. In Section 2, the basic model and notation are introduced. In Section 3. the basic idea of a probe image is presented and formally defined. Section 4 covers various algorithms that can be used to prove - in certain cases - that a given probe image cannot be present in the unknown original image. Section 5 presents a series of simulation experiments that was performed to obtain a first assessment of the capabilities of the proposed method. Conclusions are drawn in Section 6.

\section{Basic Notation and Model}

Throughout the discrete tomography literature, several imaging models have been considered: the grid model, the strip model, the line model, etc. [14, section 7.4.1]. In this paper we focus on the strip model, but our approach can be used for other projection models as well.

In the strip model, a projection is computed by considering a set of parallel strips in a given direction and for each strip computing the weighted sum of all the pixels which intersect that strip with a weight equal to the intersection area of the strip and the pixel (see Fig. 1).

We now define some general notation. An image is represented by a vector $\boldsymbol{x}=\left(x_{i}\right) \in \mathbb{R}^{n}$. We refer to the entries of $\boldsymbol{x}$ as pixels, which correspond to unit squares in the strip model. Throughout this paper we assume that all images are square, consisting of $c$ rows and $c$ columns, where $n=c^{2}$. A binary image corresponds with a vector $\overline{\boldsymbol{x}} \in\{0,1\}^{n}$. 
For a given set of $k$ projection directions, the projection map maps an image $\boldsymbol{x}$ to a vector $\boldsymbol{p} \in \mathbb{R}^{m}$ of projection data, where $m$ denotes the total number of line measurements. As the projection map is a linear transformation, it can be represented by a matrix $\boldsymbol{W}=\left(w_{i j}\right) \in \mathbb{R}^{m \times n}$, called the projection matrix. Entry $w_{i j}$ represents the weight of the contribution of $x_{j}$ to projected line $i$. Note that for the strip model its entries are real values in $[0,1]$. From this point on, we assume that the projection matrix has the property that $\sum_{i=1}^{m} w_{i j}=k$ for all $j=1, \ldots, n$. This property is satisfied for the strip projection model,

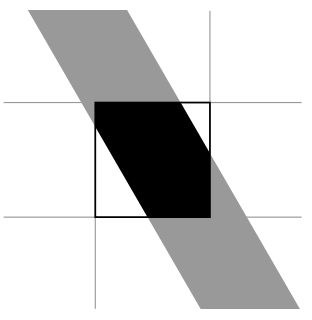

Fig. 1. The strip model as the total pixel weight for each projection angle is equal to the area of a pixel, which is 1 .

The general reconstruction problem consists of finding a solution of the system $\boldsymbol{W} \boldsymbol{x}=\boldsymbol{p}$ for given projection data $\boldsymbol{p}$, i.e., to find an image that has the given projections. In binary tomography, one seeks a binary solution of the system. For a given projection matrix $\boldsymbol{W}$ and given projection data $\boldsymbol{p}$, let $S_{\boldsymbol{W}}(\boldsymbol{p})=$ $\left\{\boldsymbol{x} \in \mathbb{R}^{n}: \boldsymbol{W} \boldsymbol{x}=\boldsymbol{p}\right\}$, the set of all real-valued solutions corresponding with the projection data, and let $\bar{S}_{\boldsymbol{W}}(\boldsymbol{p})=S_{\boldsymbol{W}}(\boldsymbol{p}) \cap\{0,1\}^{n}$, the set of binary solutions of the system. As the main goal of incorporating prior knowledge of the binary grey levels in the reconstruction is to reduce the number of required projections, we focus on the case where $m$ is small with respect to $n$, such that the real-valued reconstruction problem is severely underdetermined.

\section{Probe Structure}

We now introduce the concept of a probe image. A probe image is represented by a vector $\boldsymbol{v}=\left(v_{i}\right) \in\{0,1, ?\}^{n}$. We say that a binary image $\overline{\boldsymbol{x}}$ satisfies the probe image $\boldsymbol{v}$ iff $\bar{x}_{i}=v_{i}$ whenever $v_{i} \in\{0,1\}$. This relation is denoted by the predicate $F(\boldsymbol{v}, \overline{\boldsymbol{x}})$. In other words, the zeros and ones in the probe image prescribe the values of the corresponding pixels in $\overline{\boldsymbol{x}}$, while a pixel value of '?' in the probe image allow any pixel value in the corresponding pixel of $\overline{\boldsymbol{x}}$. We denote the set of all images satisfying a probe image $\boldsymbol{v}$ by $\mathcal{F}(\boldsymbol{v})=\left\{\overline{\boldsymbol{x}} \in\{0,1\}^{n}: F(\boldsymbol{v}, \overline{\boldsymbol{x}})\right\}$. For any probe image $\boldsymbol{v}$, define $s(\boldsymbol{v})=\#\left\{1 \leq i \leq n: v_{i} \neq^{\prime} ?^{\prime}\right\}$, the total number of 0 's and 1's in the probe image.

Suppose that we want to know if the unknown original image may contain a certain homogeneous region of 1's (i.e., white pixels). We then define a probe image $\overline{\boldsymbol{v}}$ that has such a homogeneous region, and contains the '?' symbol in all pixels that are not in this region (see Fig. 2a). The question whether there exists a binary solution of the tomography problem that has such a region can then be rephrased as a check whether the set $\bar{S}_{\boldsymbol{W}}(\boldsymbol{p}) \cap \mathcal{F}(\boldsymbol{v})$ is empty or not. Similarly, one can define an edge detection probe image such as shown in Fig. 2b. Any image that has a horizontal edge at the indicated position, consisting of a black strip of background pixels and a white strip of foreground pixels (i.e., an edge at the bottom of a white region), will be part of the set $\mathcal{F}(\boldsymbol{v})$ for this probe image $\boldsymbol{v}$. This brings us to the central problem considered in this paper: 

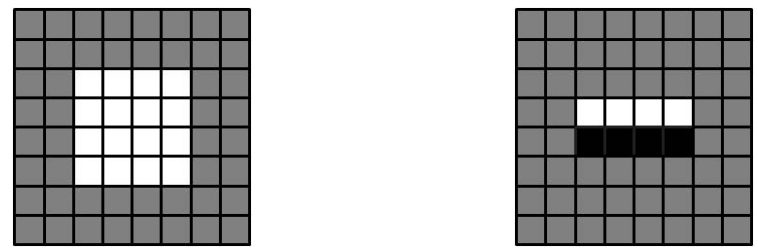

Fig. 2. Two probe images. The pixels are colored as follows: black (0), grey (?), white (1). Left: homogeneous white region; right: horizontal edge at the bottom of a white region.

Problem 1. (Probe problem). Let $\boldsymbol{W} \in \mathbb{R}^{m \times n}$ be a given projection matrix and let $\boldsymbol{v} \in\{0,1, ?\}^{n}$ be a given probe image. Determine if $\bar{S}_{\boldsymbol{W}}(\boldsymbol{p}) \cap \mathcal{F}(\boldsymbol{v})=\emptyset$.

If the intersection between the solution set of the tomography problem and the set of images that satisfy the probe image is not empty, we cannot conclude if the unknown original image satisfies the probe image. However, if the intersection between both sets is empty, we can conclude that no binary solution exists that has the probed feature. As we will see in the next sections, one can often prove that the answer to Problem[1] is "yes", even without enumerating the set $\bar{S}_{\boldsymbol{W}}(\boldsymbol{p})$ of binary solutions of the reconstruction problem.

Now consider the system of equations

$$
\left(\begin{array}{ccc}
\mid & & \mid \\
\boldsymbol{w}_{1} & \cdots & \boldsymbol{w}_{n} \\
\mid & & \mid
\end{array}\right)\left(\begin{array}{c}
x_{1} \\
\vdots \\
x_{n}
\end{array}\right)=\boldsymbol{p}
$$

where $\boldsymbol{w}_{i}$ denotes the $i$ th column vector of $\boldsymbol{W}$. We now define the operation of fixing a pixel $x_{i}$ at value $v_{i} \in \mathbb{R}$. It transforms the system (1) into the new system

$$
\left(\begin{array}{ccccc}
\mid & & & \mid & \\
\boldsymbol{w}_{1} \cdots & \boldsymbol{w}_{i-1} & \boldsymbol{w}_{i+1} & \cdots & \boldsymbol{w}_{n} \\
\mid & & \mid & & \mid
\end{array}\right)\left(\begin{array}{c}
x_{1} \\
\vdots \\
x_{i-1} \\
x_{i+1} \\
\vdots \\
x_{n}
\end{array}\right)=\boldsymbol{p}-v_{i} \boldsymbol{w}_{i}
$$

The new system has the same number of equations as the original system, whereas the number of variables is decreased by one. The fixing operation can be performed for more than one pixel at time.

Proposition 1. Let $\boldsymbol{W} \in \mathbb{R}^{m \times n}$ be a given projection matrix and let $\boldsymbol{v} \in$ $\{0,1, ?\}^{n}$ be a given probe image. Let $\boldsymbol{R} \boldsymbol{y}=\boldsymbol{q}$ be the linear system that is obtained by fixing all pixels $x_{i}$ to value $v_{i}$ whenever $v_{i} \in\{0,1\}$. Then solving Problem 1 is equivalent to checking whether $\bar{S}_{\boldsymbol{R}}(\boldsymbol{q})=\emptyset$. 
We call the linear system formed in Prop. 1 the reduced linear system corresponding to the probe image $\boldsymbol{v}$.

\section{Partially Solving the Probe Problem}

As noted in the previous section, the probe problem can be rephrased as the question whether or not the reduced linear system has a binary solution. In [13], the authors present a sufficient condition for the existence of binary solutions of a given linear system $\boldsymbol{W} \boldsymbol{x}=\boldsymbol{p}$ which satisfies $\sum_{i=1}^{m} w_{i j}=k$ for all $j=1, \ldots, n$. In summary, it is proved that all binary solutions of this linear system lie on a hypersphere centered in the minimum norm solution $\boldsymbol{x}^{*}$ and having radius $\mathcal{R}\left(\boldsymbol{p}, \boldsymbol{x}^{*}\right)=\sqrt{\frac{\sum_{i=1}^{m} p_{i}}{k}-\left\|\boldsymbol{x}^{*}\right\|_{2}^{2}}$. If the binary vector closest to $\boldsymbol{x}^{*}$ is outside this hypersphere then the given linear system contains no binary solutions:

Theorem 1. Let $\boldsymbol{x}^{*}=\boldsymbol{W}^{\dagger} \boldsymbol{p}$, where $\boldsymbol{W}^{\dagger}$ denotes the Moore-Penrose inverse of $\boldsymbol{W}[15]$. For $\alpha \in \mathbb{R}$, let $\rho(\alpha)=\min (|\alpha|,|1-\alpha|)$ and put $T\left(\boldsymbol{x}^{*}\right)=\sqrt{\sum_{i=1}^{n} \rho^{2}\left(x_{i}^{*}\right)}$. If $\mathcal{R}\left(\boldsymbol{p}, \boldsymbol{x}^{*}\right)<T\left(\boldsymbol{x}^{*}\right)$, then $\bar{S}_{\boldsymbol{W}}(\boldsymbol{p})=\emptyset$.

Proof. See [13].

In the remainder of this section we present two related techniques for proving that the unknown original image does not satisfy a given probe image $\boldsymbol{v}$. Both methods use variants of Theorem 1 .

\subsection{Probing by Analyzing the Binary Solutions of the Reduced Linear System}

Let $\boldsymbol{v}$ be a given probe image. We now analyze the reduced linear system $\boldsymbol{R} \boldsymbol{y}=\boldsymbol{q}$ corresponding to $\boldsymbol{v}$, in terms of the existence of binary solutions, following the idea of Theorem 1 .

Let $\boldsymbol{y}^{*}=\boldsymbol{R}^{\dagger} \boldsymbol{q}$ and $\rho(\alpha)=\min (|\alpha|,|1-\alpha|)$. Put $T\left(\boldsymbol{y}^{*}\right)=\sqrt{\sum_{i=1}^{n} \rho^{2}\left(y_{i}^{*}\right)}$ and define $\mathcal{T}\left(\boldsymbol{y}^{*}\right)=\left\{\overline{\boldsymbol{r}} \in\{0,1\}^{n-s(\boldsymbol{v})}:\left\|\overline{\boldsymbol{r}}-\boldsymbol{y}^{*}\right\|_{2}=T\left(\boldsymbol{y}^{*}\right)\right\}$. Also, let $\overline{\boldsymbol{r}} \in \mathcal{T}\left(\boldsymbol{y}^{*}\right)$, i.e., $\overline{\boldsymbol{r}}$ is among the binary vectors that are nearest to $\boldsymbol{y}^{*}$ in the Euclidean sense. Vector $\overline{\boldsymbol{r}}$ can be easily computed by rounding the entries of $\boldsymbol{y}^{*}$ to their nearest value in the set $\{0,1\}$. Despite $\overline{\boldsymbol{r}}$ may not be unique, any choice of $\overline{\boldsymbol{r}}$ yields the same results in this context.

Rewriting Theorem 1 in the framework of identifying the existence of binary images satisfying a given probe image $\boldsymbol{v}$, we have:

Theorem 2. Let $\boldsymbol{y}^{*}=\boldsymbol{R}^{\dagger} \boldsymbol{q}$ and $\overline{\boldsymbol{r}} \in \mathcal{T}\left(\boldsymbol{y}^{*}\right)$. If $\left\|\overline{\boldsymbol{r}}-\boldsymbol{y}^{*}\right\|_{2}>\sqrt{\frac{\sum_{i=1}^{m} q_{i}}{k}-\left\|\boldsymbol{y}^{*}\right\|_{2}^{2}}$ then the original system $\boldsymbol{W} \boldsymbol{x}=\boldsymbol{p}$ does not have a binary solution which satisfies $\boldsymbol{v}$.

Proof. From Theorem 1, we know that if $\left\|\overline{\boldsymbol{r}}-\boldsymbol{y}^{*}\right\|_{2}>\sqrt{\frac{\sum_{i=1}^{m} q_{i}}{k}-\left\|\boldsymbol{y}^{*}\right\|_{2}^{2}}$ then there is no binary vector satisfying $\boldsymbol{R} \boldsymbol{y}=\boldsymbol{p}$. Hence, there is no $\overline{\boldsymbol{x}} \in \bar{S}_{\boldsymbol{W}}(\boldsymbol{p})$ that satisfies $\boldsymbol{v}$. 


\subsection{Probing by Analyzing the Binary Solutions of the Original Linear System}

Using an idea similar to what was used in the previous subsection, we now analyze the consistency of the original linear system with respect to binary solutions. However, instead of using $\overline{\boldsymbol{r}}$, the binary vector closest to the minimum norm solution $\boldsymbol{x}^{*}$, we define $\tilde{\boldsymbol{r}}$ as the binary vector, satisfyig the probe image $\boldsymbol{v}$, which is closest to $\boldsymbol{x}^{*}$.

Theorem 3. Let $\boldsymbol{x}^{*}=\boldsymbol{W}^{\dagger} \boldsymbol{p}$ and $\overline{\boldsymbol{r}} \in \mathcal{T}\left(\boldsymbol{x}^{*}\right)$. For $i=1, \ldots, n$, define $\tilde{r}_{i}=\bar{v}_{i}$ if $v_{i} \neq^{\prime} ?^{\prime}$ and $\tilde{r}_{i}=\bar{r}_{i}$ otherwise. If $\left\|\tilde{\boldsymbol{r}}-\boldsymbol{x}^{*}\right\|_{2}>\sqrt{\frac{\sum_{i=1}^{m} p_{i}}{k}-\left\|\boldsymbol{x}^{*}\right\|_{2}^{2}}$, then $\boldsymbol{v}$ is not satisfied by any $\overline{\boldsymbol{x}} \in \bar{S}_{\boldsymbol{W}}(\boldsymbol{p})$.

Proof. The vector $\tilde{\boldsymbol{r}}$ is the binary image which contains the structure of the probe image that is closest to $\boldsymbol{x}^{*}$. If $\tilde{\boldsymbol{r}}$ is out of the hypersphere containing all binary solutions of $\boldsymbol{W} \boldsymbol{x}=\boldsymbol{p}$ (see [13]), then there is no binary image satisfying the probe image $\boldsymbol{v}$ that is on this hypersphere. Therefore $\boldsymbol{v}$ is not satisfied by any binary solution of $\boldsymbol{W} \boldsymbol{x}=\boldsymbol{p}$.

\section{Numerical Experiments}

Although the two techniques from Section 4 can detect sufficient conditions for the non-existence of binary solutions of the reconstruction problem that satisfy the given probe image, an empirical study is needed to determine the usefulness of the proposed methods for actual tomography data. A series of experiments was performed to investigate the presented method, for three different phantom images using a variable number of projections. The experiments are all based on simulated projection data obtained by computing the projections of the test images (so-called phantoms) in Fig. [3.

For the experiments, we have used probe images that only consider 0's and 1's inside a square sub-image of size $8 \times 8$ pixels. This subregion is then moved across the full image region, scanning the possible presence of the probe structure throughout the image of size $64 \times 64$ pixels.

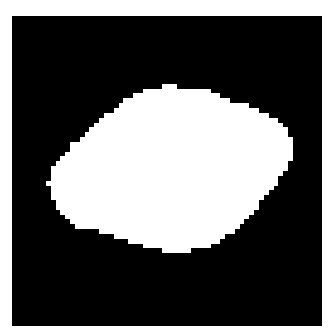

(a) Phantom 1, $64 \times 64$

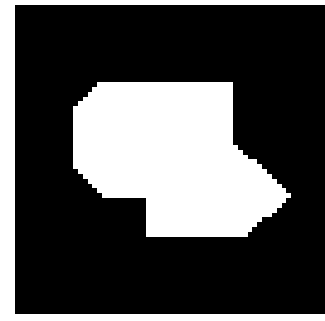

(b) Phantom 2, $64 \times 64$

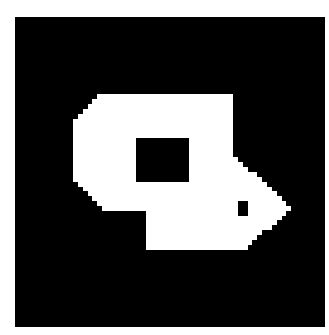

(c) Phantom 3, $64 \times 64$

Fig. 3. Original phantom images used for the experiments 
For each experiment, both techniques from Section 4 were used, checking whether any of these two methods can prove that the probe structure cannot occur in a particular region of the unknown original image.

To compute the shortest least-squares solutions of the linear systems involved in the methods of Section 4, the CGLS algorithm was used. We refer to [13] for details.

In the following subsections, we consider experiments for two different probe structures, for detecting homogeneous regions and horizontal edges, respectively.

\subsection{Homogeneous Regions}

In this section, we focus on the identification of square homogeneous regions in the unknown original image. Two types of probe images were defined: a square $8 \times 8$ region of 1 's (white pixels) surrounded by '?' pixels, and a square $8 \times 8$ region of black pixels, also surrounded by '?' pixels. These square regions were then moved across the full $64 \times 64$ image region to determine at each location whether such a homogeneous black or white square can possibly occur in the binary solution set of the tomography problem.

For each probe image we are able to define a status based on the results obtained by applying the presented methods with the two different types of probe images. We define the status forbidden for a probe image which, according to the methods, have no binary solution satisfying it. We also define the status allowed for a probe image in which the methods could not determine whether there exists a binary solution satisfying this probe image.

The results for a given phantom image leads to a new 2D greyscale image, which represents - for each position of the probe region - the outcome for both probe types, as follows:

- If the black region is allowed and the white region is forbidden then associate a black color;

- If the black region is forbidden and the white region is allowed then associate a white color;

- If the black region is allowed and the white region is allowed then associate a light grey color;

- If the black region is forbidden and the white region is forbidden then associate a dark grey color;

The resulting greyscale images are shown in Fig. 4 for Phantoms 1 and 2, depicting results for an increasing number of projection angles. It can be observed that as the number of angles grows, the results of the probe experiments provide an increasingly accurate view of the true presence of homogeneous regions in the phantom image.

\subsection{Horizontal Edges}

The goal of this section is to identify straight horizontal edges which could be present in the original image. We use the term horizontal edges to indicate 

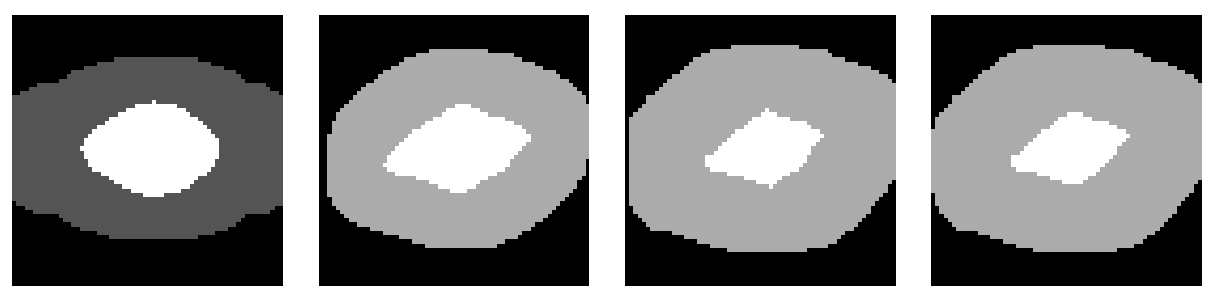

(a) Phantom 1. Number of projection angles: 2, 4, 8 and 12
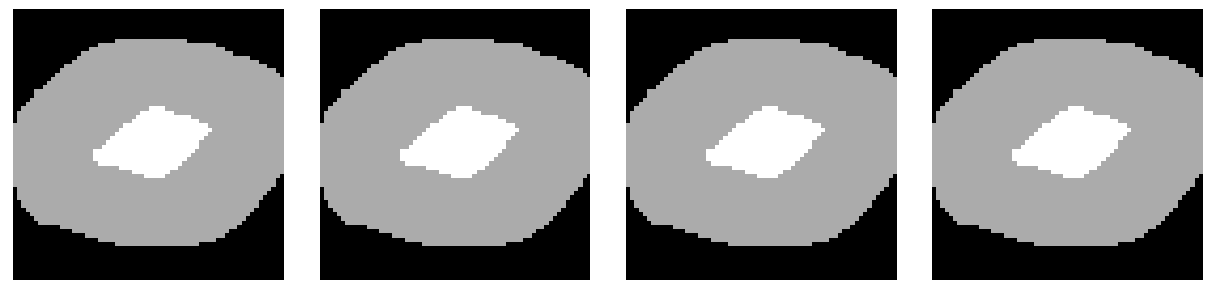

(b) Phantom 1. Number of projection angles: 16, 20, 24, and 28
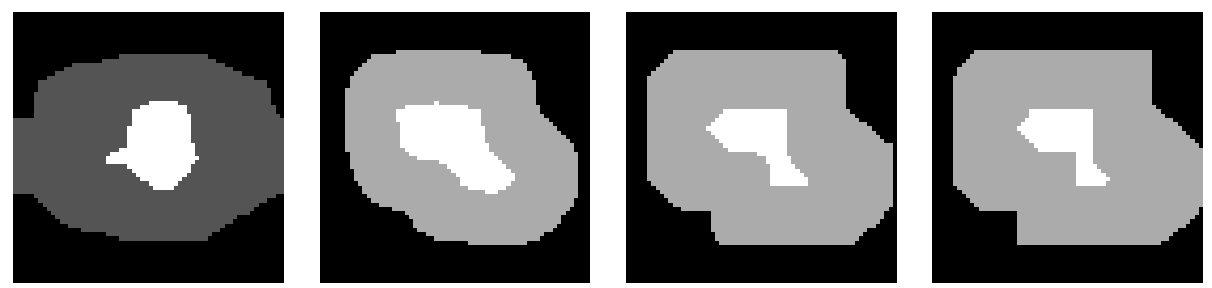

(c) Phantom 2. Number of projection angles: 2, 4, 8 and 12
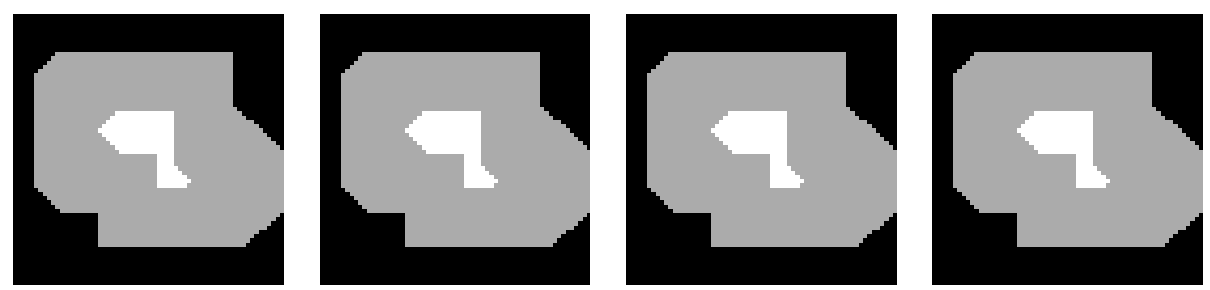

(d) Phantom 2. Number of projection angles: 16, 20, 24, and 28

Fig. 4. Homogeneous region status for the phantom images of dimension $64 \times 64$

horizontally adjacent pixels with intensity 1 (white) which are vertically adjacent to the same number of horizontally adjacent pixels with intensity 0 (black color). So, we define a square probe structure of size $8 \times 8$ such that the pixels in the upper half of the square are set to 0 and the pixels in the lower half of the square are set to 1 . The vertically mirrored version of this probe structure was also used to detect edges at the bottom of an object. 
Similar to the previous section, the results for this probe structure give rise to a new greyscale image, defined as follows. Starting from a completely black image, if at a certain position for the probe structure no unsatisfiability is detected, the "white" part of the edge (corresponding to the interior of the object) is colored white in the output image if it is also white in the original image and dark grey if it is black in the original image. The "black" part of the edge (corresponding to the outside of the object) is colored black in the output image if it is also black
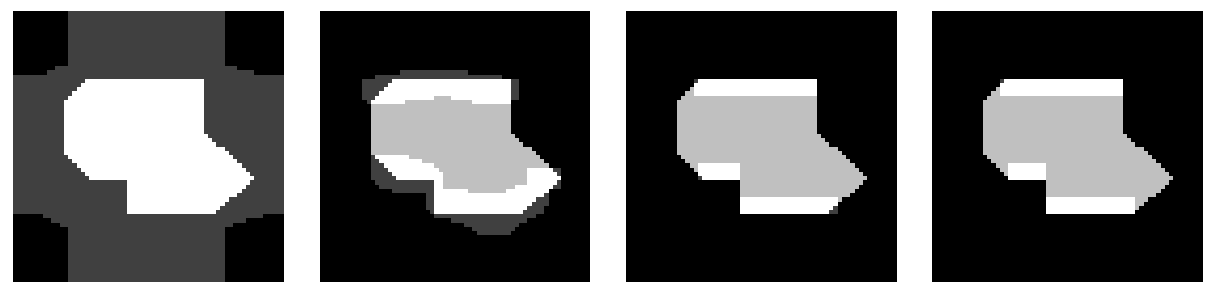

(a) Phantom 2. Number of projection angles: 2, 4, 8 and 12
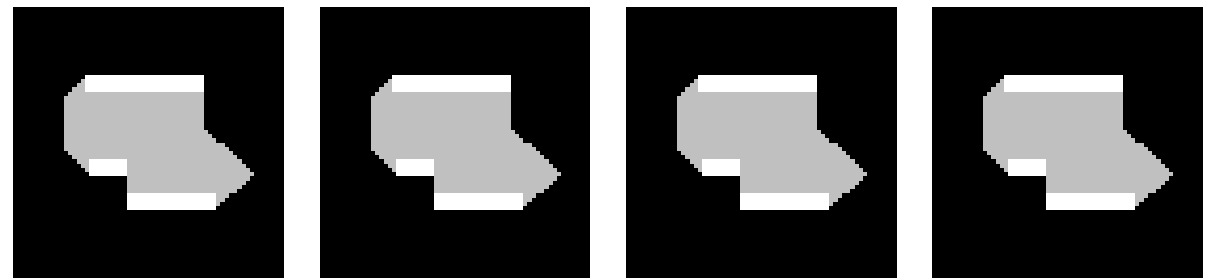

(b) Phantom 2. Number of projection angles: 16, 20, 24, and 28
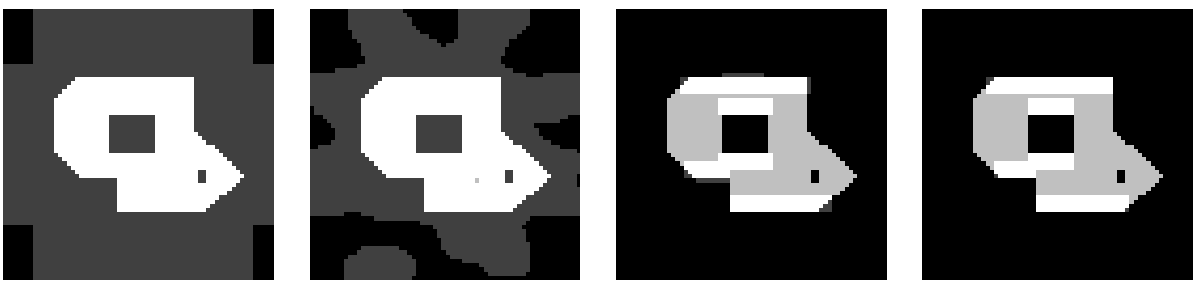

(c) Phantom 3. Number of projection angles: 2, 4, 8 and 12
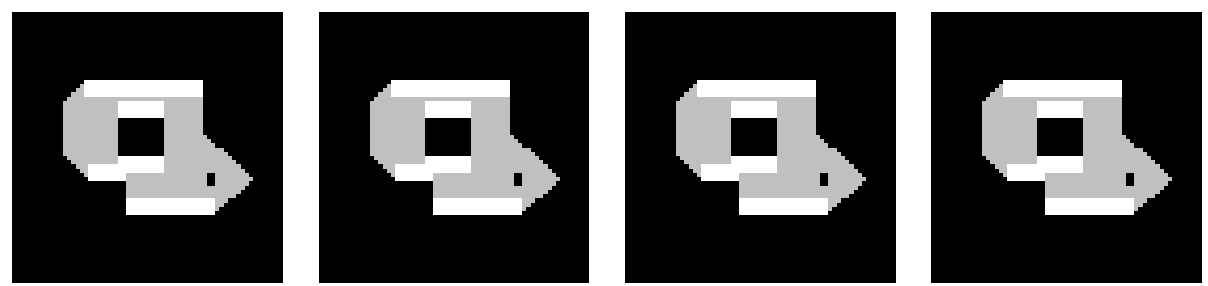

(d) Phantom 3. Number of projection angles: 16, 20, 24, and 28

Fig. 5. Possible edges for the phantom images of dimension $64 \times 64$ 
in the original image and light grey if it is white in the original image. Also, if at a certain position for the probe structure the unsatisfiability is detected but there are white pixels in this region in the original image, then those pixels are colored as light grey. The results of this procedure are shown in Fig. 5, which identifies the regions that could be edges according to our results, for a varying number of projection angles. Again, we see that as the number of projections increases, the results of the probe experiments provide an increasingly accurate view of the true presence of horizontal edges in the phantom image.

\section{Outlook and Conclusion}

In this article we have proposed a novel approach for obtaining information about an object from a small number of its projections. By using necessary conditions for the existence of binary solutions of the tomography problem, and combining these with probe images for particular substructures of the image, it can be determined whether such a substructure can possibly occur, or whether it can certainly not occur in the unknown original image.

The experimental results for a limited set of simulation experiments show that this approach can indeed lead to the recovery of substantial information about the original image, without resorting to a particular, possibly non-unique reconstruction.

More research in this direction will be necessary to determine what the limitations are of the proposed method, and how it compares to image analysis algorithms that try to find the structure directly in a reconstructed image.

Acknowledgements. W.F. acknowledges support from the Erasmus Mundus program of the European Union, and from the University of Leiden. K.J.B. was supported by the Netherlands Organisation for Scientific Research (NWO), programme 639.072.005.

\section{References}

1. Batenburg, K.J.: A network flow algorithm for reconstructing binary images from continuous X-rays. J. Math. Im. Vision 30(3), 231-248 (2008)

2. Batenburg, K.J., Sijbers, J.: Dart: a practical reconstruction algorithm for discrete tomography. IEEE Trans. Image Processing 20(9), 2542-2553 (2011)

3. Herman, G.T.: Fundamentals of Computerized Tomography: Image reconstruction from projections. Springer (2009)

4. Schüle, T., Schnörr, C., Weber, S., Hornegger, J.: Discrete tomography by convexconcave regularization and D.C. programming. Discr. Appl. Math. 151, 229-243 (2005)

5. Alpers, A., Gritzmann, P.: On stability, error correction, and noise compensation in discrete tomography. SIAM Journal on Discrete Mathematics 20(1), 227-239 (2006)

6. Alpers, A., Brunetti, S.: Stability results for the reconstruction of binary pictures from two projections. Image and Vision Computing 25(10), 1599-1608 (2007) 
7. Van Dalen, B.: Stability results for uniquely determined sets from two directions in discrete tomography. Discrete Mathematics 309, 3905-3916 (2009)

8. Hajdu, L., Tijdeman, R.: Algebraic aspects of discrete tomography. J. Reine Angew. Math. 534, 119-128 (2001)

9. Herman, G.T., Kuba, A. (eds.): Discrete Tomography: Foundations, Algorithms and Applications. Birkhäuser, Boston (1999)

10. Gara, M., Tasi, T.S., Balazs, P.: Learning connectedness and convexity of binary images from their projections. Pure Math. Appl. 20(1-2), 27-48 (2009)

11. Helgason, S.: The Radon transform. Birkhäuser, Boston (1980)

12. Stolk, A., Batenburg, K.J.: An algebraic framework for discrete tomography: Revealing the structure of dependencies. SIAM J. Discrete Math. 24(3), 1056-1079 (2010)

13. Batenburg, K.J., Fortes, W., Hajdu, L., Tijdeman, R.: Bounds on the Difference between Reconstructions in Binary Tomography. In: Debled-Rennesson, I., Domenjoud, E., Kerautret, B., Even, P. (eds.) DGCI 2011. LNCS, vol. 6607, pp. 369-380. Springer, Heidelberg (2011)

14. Kak, A.C., Slaney, M.: Principles of Computerized Tomographic Imaging. SIAM (2001)

15. Björck, A.: Numerical methods for least square problems. SIAM, Linköping University, Sweden (1996) 